\title{
Pedestrian Tracking Based on Improved Particle Filter Under Complex Background
}

\author{
$\mathrm{Yu} \mathrm{Fu}$ \\ College of Information Science \& Technology \\ Hainan University \\ Haikou,570000,China \\ E-mail:xueqiqi2011@foxmail.com
}

\author{
Xiang Long \\ College of Information Science \& Technology \\ Hainan University \\ Haikou,570000.China \\ E-mail:longxiangalpha@126.com
}

\begin{abstract}
When tracking a moving human target,the traditional particle filter algorithm based on color characteristic can't get accurate results in situations like complicated background or frequent brightness change.Due to the problem, this paper put forward a particle filter algorithm based on combination of color characteristics and shape features of the target.Firstly, fusing the above-mentioned two features into the particle filter frame to calculate the particle weights and achieve the human tracking goal through image sequences. The experimental results show that the algorithm can improve the traditional tracking algorithms based on single color feature limitations.And greatly improves the accuracy and effectiveness of the human tracking under complex background.(Abstract)
\end{abstract}

Keywords-component; Particle filter;Color feature;Shape feature; Joint similarity (key words)

\section{I NTRODUCTION}

As a core problem in computer vision community,moving targets tracking has got extensive and in-depth research.There are three main algorithms for human body tracking algorithm, Calman filtering , Meanshift algorithm, and template matching method,etc.Template matching method needs complex calculation and cannot extracte target absolutely ${ }^{[1]}$,Meanshift algorithm has fast tracking speed, but it is easy to fall into local optimum $^{[2]}$, and Calman filtering needs the target state satisfied with Gauss distribution,otherwise it will needs a large amount of calculation,even worse failure to tracking when facing the non Gauss and nonlinear problem caused by complex background or target occlusion $^{[3]}$.In recent years,particle filter algorithm developed rapidly in target tracking field,and has become a hot topic in the research of tracking target field for it's ability to deal with nonlinear, non Gauss problem ${ }^{[4,5]}$.

This paper made a research to video human body movements target tracking algorithm by using the color characteristics and the shape features of the target .The algorithm uses a combination between the feature of color histogram and the feature of edge direction histogram , firstly extract the targets' shape and color characteristic ,then combined it with the particle filter to complete the human body robust tracking in image sequences. The experimental results show that The algorithm can better adapt to the human body target tracking difficulty, light changing and background interference,etc.And improved the limitation of the traditional tracking algorithm based on color features. Of course, improved the reliability of the tracking algorithm at the same time.

\section{PARTICLE FILTER ALGORITHM}

The basic principle of particle filter is the order.importance sampling principle.Finding a group of transmitted random samples existed in the state space to approximate the probability density function $p\left(x_{k} \mid z_{k}\right)$, and using the sample mean to instead the integral operation to obtaining the process of state minimum variance estimation,then the sample is referred to as "particle".In a stationary random process, we assume the posterior probability density is $p\left(x_{k-1} \mid z_{k-1}\right)$ at k-1 moment, and then select $\mathrm{N}$ random samples according to some principles, update the state time process after obtained the measuring information at $\mathrm{K}$ moment,then the posteriori probability density of $\mathrm{Z}$ particles can be approximated as $p\left(x_{k} \mid z_{k}\right)$.With the increasing number of particles, the particle probability density function will gradually approaching the state probability density function estimation, that is, the particle filter can achieve the optimal Bayesian estimation results.

The basic particle filter algorithm often collects samples $x_{0}^{i}$ from the prior probability distribution $p\left(x_{0}\right), \mathrm{i}=1, \ldots, \mathrm{N}$. And collects samples $x_{k}^{i}$ from the reference distribution $q\left(x_{k} \mid x_{k-1}, z_{k}\right)$ at $\mathrm{K}$ moment,Simultaneously,calculates the normalized weights $w_{k}^{i}$.

$$
\begin{aligned}
& \tilde{w}_{k}^{i}=\tilde{w}_{k-1}^{i} \frac{p\left(z_{k} \mid x_{k}^{i}\right) p\left(z_{k} \mid x_{k-1}^{i}\right)}{q\left(x_{k}^{i} \mid x_{k-1}^{i}, z_{k}\right)} \\
& w_{k}^{i}=\frac{\widetilde{w}_{k}^{i}}{\sum_{j=1}^{N} \tilde{w}_{k}^{i}}
\end{aligned}
$$


Then, resamples the particle, using new samples $\left\{x_{k}^{i *}\right\}_{i=1}^{N} \quad$ to instead $\left\{x_{k}^{i}\right\}_{i=1}^{N}$, which also meet the distribution $\quad p\left\{x_{k}^{i^{*}}=x_{k}^{i}\right\}=w_{k}^{i} \quad$, and update weights $w_{k}^{i}=N^{-1}$ simultaneously, finally output the approximate posterior probability density $x_{k}: p\left(x_{k} \mid z_{1: k}\right)=\frac{1}{N} \sum_{j=1}^{N} \delta\left(x_{k}-x_{k}^{i}\right)$.After several recursives, the weight will gather in a few or even a particle,that is to say, particle degeneracy phenomenon arising, Thus failing to approach the state posterior probability density . For the core idea of resampling is diminish the lower weight particle and focusing on process high weights particles,it solved the particle degeneracy problem effectively.

\section{IMPROVED ALGORITHM FOR TRACKING}

\section{BASED ON COLOR FEATURE AND SHAPE FEATURE}

In the target tracking algorithm Based on the particle filter ,the selection of target template is an important factor in affecting the target tracking's performance.The target template refers to the target feature sets, the target based on visual image has many characteristics,such as color feature, texture feature, shape feature,etc. The traditional particle filter algorithm based only on color feature, it is not stable and is easily fail to track once targets in complex background.Therefore, in order to improve the robustness of the tracking algorithm, this paper adopts human targets color features and made a fusion with a new shape characteristics of the target to jointly establish observation model. The experimental results show that the particle filter algorithm based on characteristics fusion can reduce the noise influence effectively and increase the target tracking ability in complex background.

\section{A. Model of histogram of edge direction for tracking}

Histogram of edge direction is a statistical distribution of expressing the target object edge points' edge direction. It has been used to describe the expression of target objects ${ }^{[6]}$ and has been proved with fast computation and robust characteristics.

Hstogram of $\mathrm{M}$ dimension of a two value edge direction image is defined as follows:

$$
E_{i}=P[O(p)=i] \quad i=1, \ldots, m
$$

Among them, the direction of space is divided into $\mathrm{m}$ different directions, $\mathrm{P}$ stands for each edge point, $\mathrm{O}(\mathrm{P})$ stands for edge direction, and Ei shows all points' proportion of $i$ edge direction,the sum proportion of all $\mathrm{m}$ directions is 1 .

1) Expression of histogram of edge direction

This paper uses the Canny edge operator ${ }^{[7]}$ to calculate the binary image edge points figure and the value of every edge directions of the binary image.Then counts the proportion of each edge direction to get the histogram.In particle filter algorithm,we can hypothesis the center position of the reference target as $x_{0}, x_{i}$ stands for one point of the target area.The target template edge direction histogram can be expressed as:

$$
q_{u}=\alpha \sum_{i=1}^{N} k\left\|\frac{x_{0}-x_{i}}{h}\right\|^{2} \delta\left[O\left(x_{i}-u\right)\right]
$$

In the above formula, $\mathrm{K}$ represents an isotropic kernel function,it will give less weight to the point which are far from the object center, while the nearly point will be given a larger weight. And it will limit the points which needed to calculate in a local window, the weight of the outside points is $0, \delta(\cdot)$ is delta function. $N$ represents the whole pixels in tracking window, m represents the orientation dimension of the edge direction histogram, $\alpha$ represents the normalized parameter. Similarly, we can get the candidate target' s edge direction histogram of current frame center position yi like:

$$
p_{u}\left(y_{i}\right)=\beta \sum_{i=1}^{N} k\left\|\frac{x_{0}-x_{i}}{h}\right\|^{2} \delta\left[O\left(x_{i}-u\right)\right] \quad u=1, \ldots, m
$$

Among the above formula, h represents the size of 2$\mathrm{D}$ search window, $\beta$ represents the normalized parameter.we use Bhattacharyya coefficients ${ }^{[8]}$ to measure the similarity between the candidate target and target template:

$$
\rho\left(q_{u}, p_{u}\left(y_{i}\right)\right)=\sum_{u=1}^{m} \sqrt{q_{u} p_{u}\left(y_{i}\right)}
$$

The larger of $\rho$ calculation results, the more similar of the two target, that is, the greater the probability of candidate target is the real target.Thus, we can get the observed probability model of the boundary features

$$
p_{\text {EOH }}(z \mid x)=\frac{1}{\sqrt{2 \pi} \sigma} \exp \left(-\frac{1-\rho\left(q_{u}, p_{u}\left(y_{i}\right)\right)}{2 \sigma^{2}}\right)
$$

Among the above formula, $\sigma$ is an experience constant which can be selected according to the tracking performance ${ }^{[9]}$.

\section{B. Color histogram model for tracking}

The general particle filter algorithm for tracking target using color histogram to describe the target's appearance.Divide each subspace of the RGB color space into k equal interval,each section is called a bin characteristic space,the number of the characteristic value $u$ is $m_{c}=k^{3}$,Calculate each feature value's probabilities to all pixels of the image in target area.The statistical histogram is the color feature description of the target.Due to the influce of occlusion or background, pixels near the center of the target model are more reliable than the periphery pixels, and has bigger identify value;therefore, system will give a weight to each pixel in the region while calculating the color characteristic value, a large weight to the pixel which near the center , and a small weight which is far away from the center,as a result, the probability density of the target characteristic value $u=1 \ldots m_{c}$ is : 


$$
\widehat{q}_{c_{u}}=C \sum_{n=1}^{N} k\left\|\frac{x_{0}-x_{i}}{h}\right\|^{2} \delta\left[c\left(x_{i}-u\right)\right]
$$

In the above formula, $x_{0}$ represents the target area's center, $\left\{x_{i} \mid i=1,2, \ldots n\right\}$ represents $n$ pixels in the area.K ( ) is defined as the kernel function's profile function of each pixel value. The function of the formula $\left\|\frac{x_{0}-x_{i}}{h}\right\|$ is to remove the impact of different size objects while calculating.h represents Kernel bandwidth, $\delta(x)$ is a delta function. $\delta\left[c\left(x_{i}\right)-u\right]$ is to determine whether the color value of the pixel xi in the target area is equal to the $\mathrm{u}$ bin. If true,1,or $0 . \mathrm{C}$ is a standardized constant coefficient which need to made $\sum_{u=1}^{m} q_{c_{u}}=1$, so $C=\frac{1}{\sum_{i=1}^{n} k\left(\left\|\frac{x_{0}-x_{i}}{h}\right\|^{2}\right.}$. Type (8) is the kernel density estimation expression based on the kernel function's profile function.

In the second frame of the sequence image and the later frame area that may contains the target is called the candidate region, the center coordinates $\mathrm{Y}$ is also the center coordinates of kernel function,pixels in the region is represented as $\left\{x_{i} \mid i=1,2, . ., n\right\}$, the probability density of the Color characteristic value of the candidate regions is :

$$
\hat{p}_{c_{u}}(y)=C_{h} \sum_{i=1}^{n_{h}} k\left\|\frac{y-x_{i}}{h}\right\|^{2} \delta\left[c\left(x_{i}-u\right)\right]
$$
coefficient.

Using Bhattacharyya coefficient to measure the similarity between the candidate goals and objectives template:

$$
\rho\left(\widehat{q}_{c_{u}}, \hat{p}_{c_{u}}(y)\right)=\sum_{u=1}^{m_{c}} \sqrt{\hat{q}_{c_{u}} \hat{p}_{c_{u}}(y)}
$$

The corresponding observation probability model of the color features is:

$$
p_{\text {colour }}(z \mid x)=\frac{1}{\sqrt{2 \pi} \lambda} \exp \left(-\frac{1-\rho\left(\widehat{q}_{c_{u}} \hat{p}_{c_{u}}(y)\right)}{2 \lambda^{2}}\right)
$$

In the above formula, $\lambda$ is a empirical constant.

\section{Implementation method for improved particle filter algorithm}

After description of a target characterization and candidate characteristics,system uses the similarity function to measure the similarity between the initial target description and the candidate target description,and get the similarity function maximized by searching the current frame to get the location of the object, so as to achieve the purpose of tracking.

The concrete realization process of the algorithm for tracking a moving human target based on color features and shape features showed as follows:firstly,draw the target template from initial frame with the mouseto get the initial state of the target parameters $\left(x_{1}, y_{1}, \dot{x}_{1}, \dot{y}_{1}\right),\left(x_{1}, y_{1}\right)$ represents the location of the target center $\left(\dot{x_{1}}, \dot{y}_{1}\right)$ represent the target speed;system random distribution of particles set $s_{1}=\left\{\left(\left(x_{1}^{i}, y_{1}^{i}, \dot{x}_{1}, \dot{y}_{1}\right), w_{1}^{i}\right)\right\}_{i=1}^{N}$ in the vicinity of the target initial state, and $\left\{w_{1}^{i}=1 / N\right\}_{i=1}^{N}, \mathrm{~N}$ is the number of particles.Then, use the autoregressive model to get the state ransition 's later particle set

$$
s_{k}-s_{k-1}=s_{k-1}-s_{k-2}+r * u_{k-1}
$$

In the above formula, $r=\frac{\sqrt{N}}{2}$ stands for the particle's random spread adius; $u_{k-1}$ is random number in[-1,1].

When calculating the particle weights, it is not only need to take advantage of its color characteristics, but also combined with the shape feature.Thus,getting the joint particle filter observation model

$$
p\left(z_{k} \mid s_{k}^{(i)}\right)=\eta \cdot p_{\text {colour }}(z \mid x)+(1-\eta) \cdot p_{E O H}(z \mid x)
$$

In the above formula, $\eta \in[0,1]$ is used to adjust the proportion of each characteristic 's observation probability over the total observation probability. When the difference of color between target and background is big, $\eta$ get larger values. If the color of the target is similar to the background, $\eta$ get smaller values.

Particle weights of feature fusion is:

$$
w_{k}^{(i)}=w_{k-1}^{(i)} p\left(z_{k} \mid s_{k}^{(i)}\right) \quad \mathrm{i}=1, \ldots, \mathrm{N}
$$

After we calculate the particle weights, normalize the weights, and use resampling technique to avoid degradation.Finally, we can calculate the target state results of the current frame by the sum of the weighted particle :

$$
\hat{s}_{k}=E\left(s_{k} \mid z_{1: k}\right)=\sum_{i=1}^{N} w_{k}^{(i)} x_{k}^{(i)}
$$

In order to further improve the robustness of the algorithm,this paper uses update policy of the reference target's color model while tracking, we assume that the average state of the target is $\hat{s}_{k}$ on $\mathrm{K}$ moment. The color histogram it corresponds to is $p_{c}\left(\hat{S}_{k}\right)$. In order to avoid the target is occluded unnecessary model updates, we need to set the 
reference target update conditions of the feature color model ,that is, the reference target's color distribution and the Bhattacharyya coefficient must greater than the preset threshold th.on this basis, we define the color characteristics 'updating formula of the reference target

$$
q_{u, k}=\left\{\begin{array}{l}
(1-\omega) \cdot q_{u, k-1}+\omega p_{u}\left(\hat{s}_{k}\right), \rho\left(q_{u, k-1}, p_{u}\left(\hat{s}_{k}\right)>t h\right. \\
q_{u, k-1}, \text { therwise }
\end{array}\right.
$$

In the above formula, $\omega \in[0,1]$ is represented the contribution of $p_{u}\left(\hat{s}_{k}\right)$ on the reference model.In order to prevent excessive updates of the reference target color model. $\omega$ generally take a very small value.

\section{ANALYSIS OF EXPERIMENTAL RESULTS}

The system is programmed in the Visual studio2008 integrated development environment , and .And it is able to track the moving human target accurately based on the OpenCV ${ }^{[10]}$ and MATLAB.In order to prove the effectiveness of the proposed algorithm.Experiment is done in two situations,one is the target color similar to background while the other one is light changes often.In both scenarios,the system use this algorithm and the traditional algorithm to track a moving human target in the video image sequence.And we made a comparation and analysis with the tracking results of these two algorithms.

\section{A. The tracking results of the target color similar to background}

This experiment uses Video sequence of outdoor complex background taken by the author the number of the frames is a total of 350 , the following figures are the results of the two algorithms for tracking.

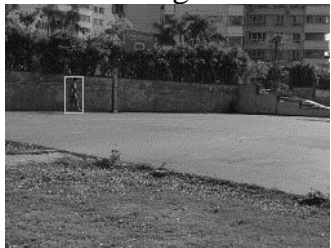

frame 8

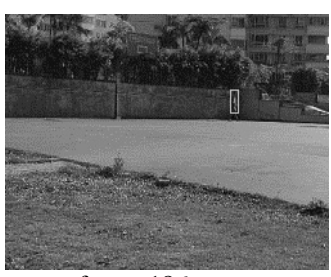

frame 186

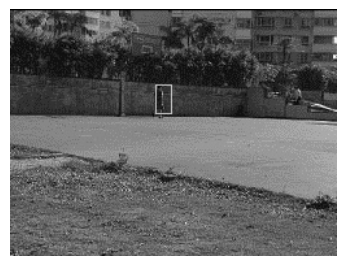

frame 59

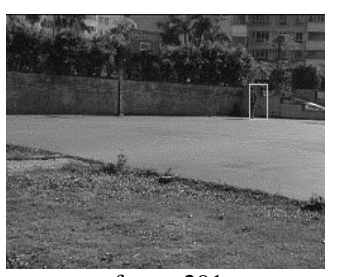

frame 291
Figure 1. Algorithm based on color feature

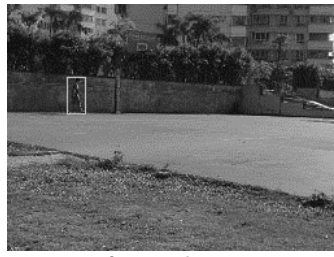

frame 8

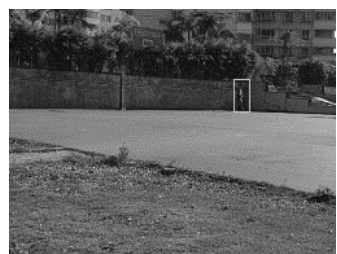

frame 186

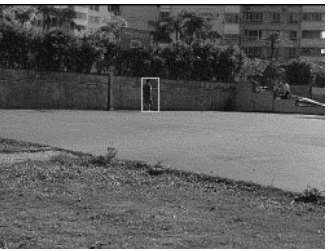

frame 59

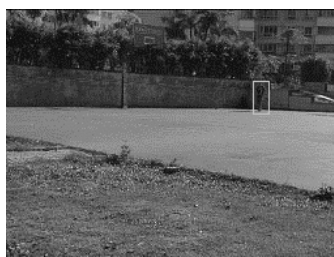

frame 291
Figure 2. Improved particle filter algorithm

As is shown in Figure 1 and Figure 2,the color of the human target wearing a gray suit is similar to the background ,Figure 1 is used the traditional particle filter algorithm, the result has a great error. While Figure 2 shows an accurate tracking result. Which shows that the improved algorithm proposed in this paper can overcome the similar color between objectives and background.

\section{B. The results of tracking in background light-changing interference}

Experiment used a video sequence of the target pedestrians from shade to bright light,the number of the frames is a total of 300,Figure 3 and Figure 4 are the results of the two algorithms for tracking.

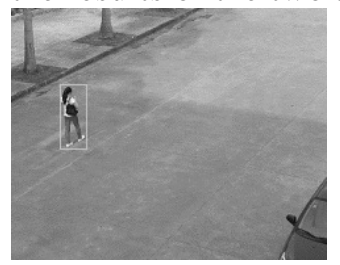

frame 5

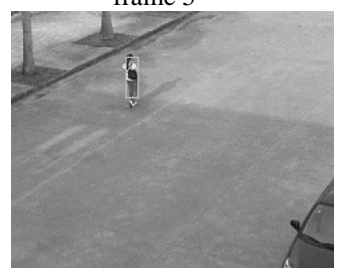

frame 193

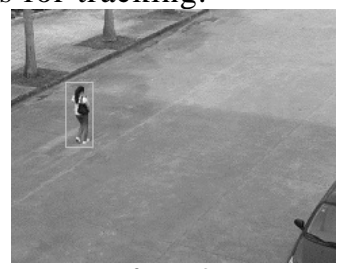

frame 36

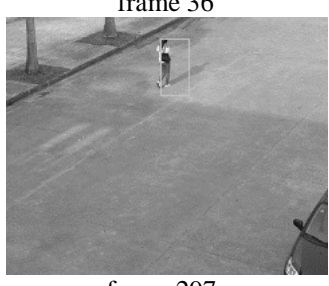

frame 297
Figure 3. Algorithm based on color feature 


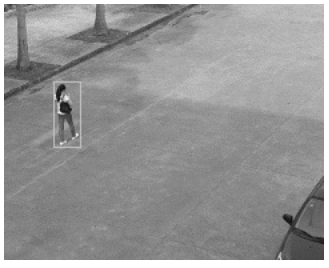

frame 5

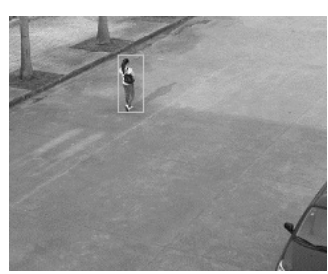

frame 193

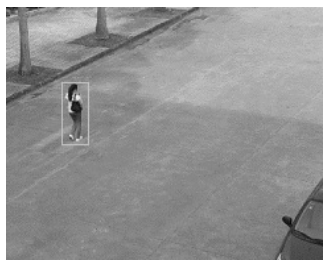

frame 36

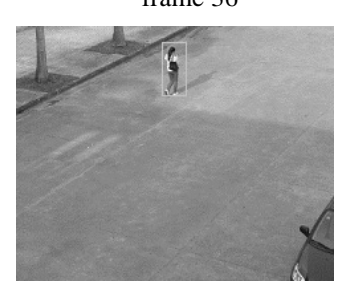

frame 297
Figure 4. Improved particle filter algorithm

Figure 3 and Figure 4 show the tracking result of the light changes in the background. The human target walks from the shadows to the light.Figure 3 shows that the result based on the traditional particle filter algorithm is with a great deal of instability in the obvious light changes conditions,.Figure 4 indicate that improved particle filter algorithm can get a very good tracking result.

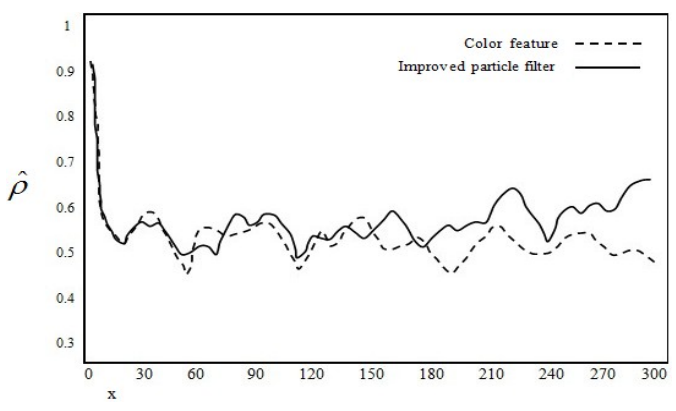

Figure 5. Joint similarity of color similar

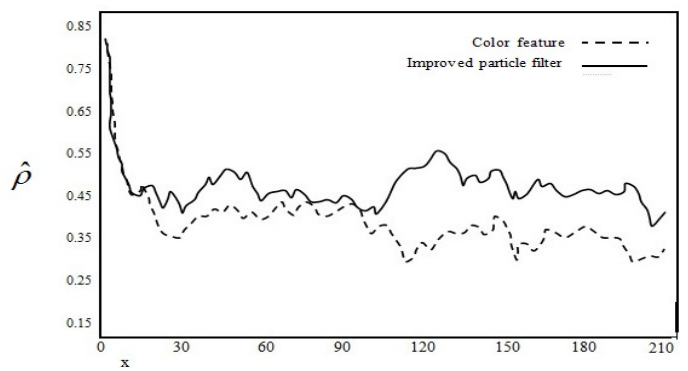

Figure 6. Joint similarity of light change

Figure 5 and Figure 6 show the joint similarity's statistical results for each frame of of the tracking process.And the abscissa $\mathrm{x}$ is the post sequence.From which we can find that:the tracking results of improved particle filtering algorithm and the similarity of the Joint initial area is superior to the traditional particle filter algorithm based on color feature.Therefore, the improved algorithm has better performance for it can overcome the background's silimar color interferenceand light change interference in target tracking process, which ensured the reliability .

\section{CONCLUSIONS}

As an new study field,the human body tracking technology in the video image sequence has a prospect application in military and civilian.In order to solve the limitations of the classical particle filter algorithm when tracking a moving human target, this paper propose a new particle filter algorithm, which based on multiple characteristics. This algorithm combines the color and shape features and it is able to track target effectivly ,accurately under complex conditions.Experimental results show that the algorithm can better adapt to the interference of the background like light changes, silimar colour and so on.The new algorithm has broken through the limitations of the traditional particle filter,meanwhile,it has improven the robustness of the algorithm for tracking moving target.

In the future research,we will further improve the algorithm,it will put forward a broader applicability, reliability and better method for tracking in the target block, multi-objective case.

\section{ACKNOWLEDGMENT}

This work is supported by Program of International S\&T Cooperation[GJXM20100002], Hainan Province Natural Science Fund[808132], Scientific Research in Higher Education Department of Hainan Province [Hjkj2011-08] and 211 Project of Hainan University.

\section{REFERENCES}

[1] Yasuno M, Ryousuke S, Yasuda N, et al. Pedestran detection and tracking in far infrared images [C]// Proc. of IEEE Intelligent Transportation Systems, 2005. USA: IEEE, 2005: 182-187.

[2] Binelli E, Broggi A, Fascioli A, et al. A modular tracking system for far infrared pedestrian recognition [C]// Proc. of IEEE Intelligent Vehicles Symposium, 2005. USA: IEEE, 2005: 759-764..

[3] Fengliang $\mathrm{Xu}$, Xia Liu, Fujimura K. Pedestrian detection and tracking with night vision [J]. IEEE Trans. on Intelligent Transportation Systems (S1524-9050), 2005, 6(1): 63-71. Systems (S1524-9050), 2005, 6(1): 63-71.

[4] LI Jin, YU Hong, ZHOU Lu-lu, et al. Human Motion Tracking Based on Quantum Genetic and Unscented Particle Filter [J]. Journal of System Simulation (S1004-731X), 2008, 20(18): 4867-4871.

[5] S J McKenna, H Nait-Charif. Tracking human motion using auxiliary particle filters and iterated likelihood weighting [J]. Image and Vision Computing (S0262-8856), 2007, 25(6): 852-862.

[6] Zhu W,Levinson S E Edge orientation-based multiview object recognition [C]//ICPR00 Barcclona: IEEE CS Press.2000:19361939.

[7] McInerney $\mathrm{T}$.Terzopou Ios D. T-snakes: Topology adaptive snakes[J]. M ed ical Image A nalysis, 2000, 2: 73- 9L. 
[8] NUMMIARO K, KOLLER-MEIER E, GOOL L V. An adaptive color-based particle filter[J]. Image and Vision Computing, 2003, 21 (1) : 99-110.
[9] Perez P, Hue C, Vermaak J, et al. Color-based probabilistic tracking $[C] / /$ Proc. of 7th European Conference on Computer Vision, Copenhagen, Denmark. USA: IEEE, 2002, 1: 661-675.

[10] BRADSKI G,KAEBLER A . Learning OpenCV[M] . Beijing: Tsinghua University Press, 2009. 